
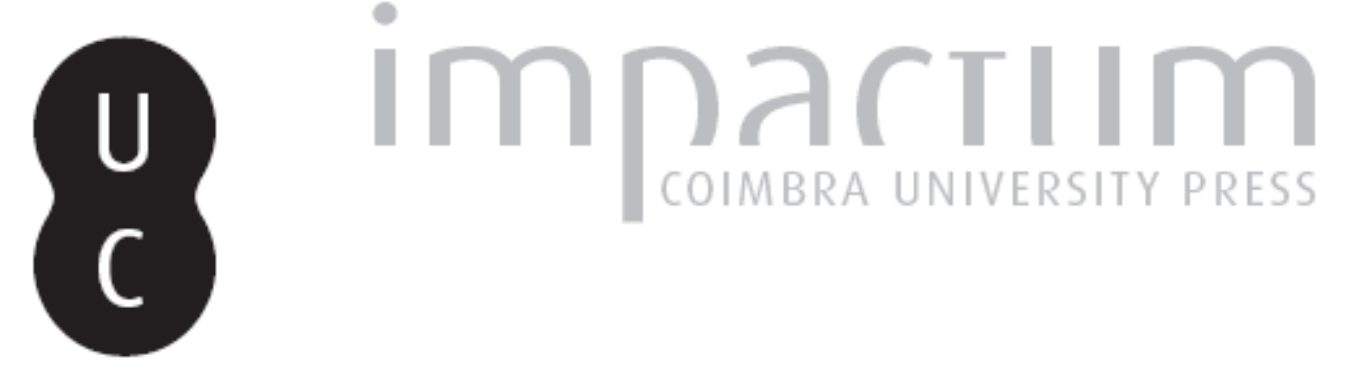

\title{
Sistema de gestão de riscos viários com o uso da geointeligência: os deslizamentos e as inundações em rodovias do Estado de Santa Catarina - Sul do Brasil
}

Autor(es): Diesel, Lilian Elizabeth

Publicado por: Associação Portuguesa de Riscos, Prevenção e Segurança

URL

persistente:

URI:http://hdl.handle.net/10316.2/35840

DOI:

DOI:http://dx.doi.org/10.14195/1647-7723_20_7

Accessed : $\quad$ 26-Apr-2023 10:37:58

A navegação consulta e descarregamento dos títulos inseridos nas Bibliotecas Digitais UC Digitalis, UC Pombalina e UC Impactum, pressupõem a aceitação plena e sem reservas dos Termos e Condições de Uso destas Bibliotecas Digitais, disponíveis em https://digitalis.uc.pt/pt-pt/termos.

Conforme exposto nos referidos Termos e Condições de Uso, o descarregamento de títulos de acesso restrito requer uma licença válida de autorização devendo o utilizador aceder ao(s) documento(s) a partir de um endereço de IP da instituição detentora da supramencionada licença.

Ao utilizador é apenas permitido o descarregamento para uso pessoal, pelo que o emprego do(s) título(s) descarregado(s) para outro fim, designadamente comercial, carece de autorização do respetivo autor ou editor da obra.

Na medida em que todas as obras da UC Digitalis se encontram protegidas pelo Código do Direito de Autor e Direitos Conexos e demais legislação aplicável, toda a cópia, parcial ou total, deste documento, nos casos em que é legalmente admitida, deverá conter ou fazer-se acompanhar por este aviso.

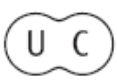




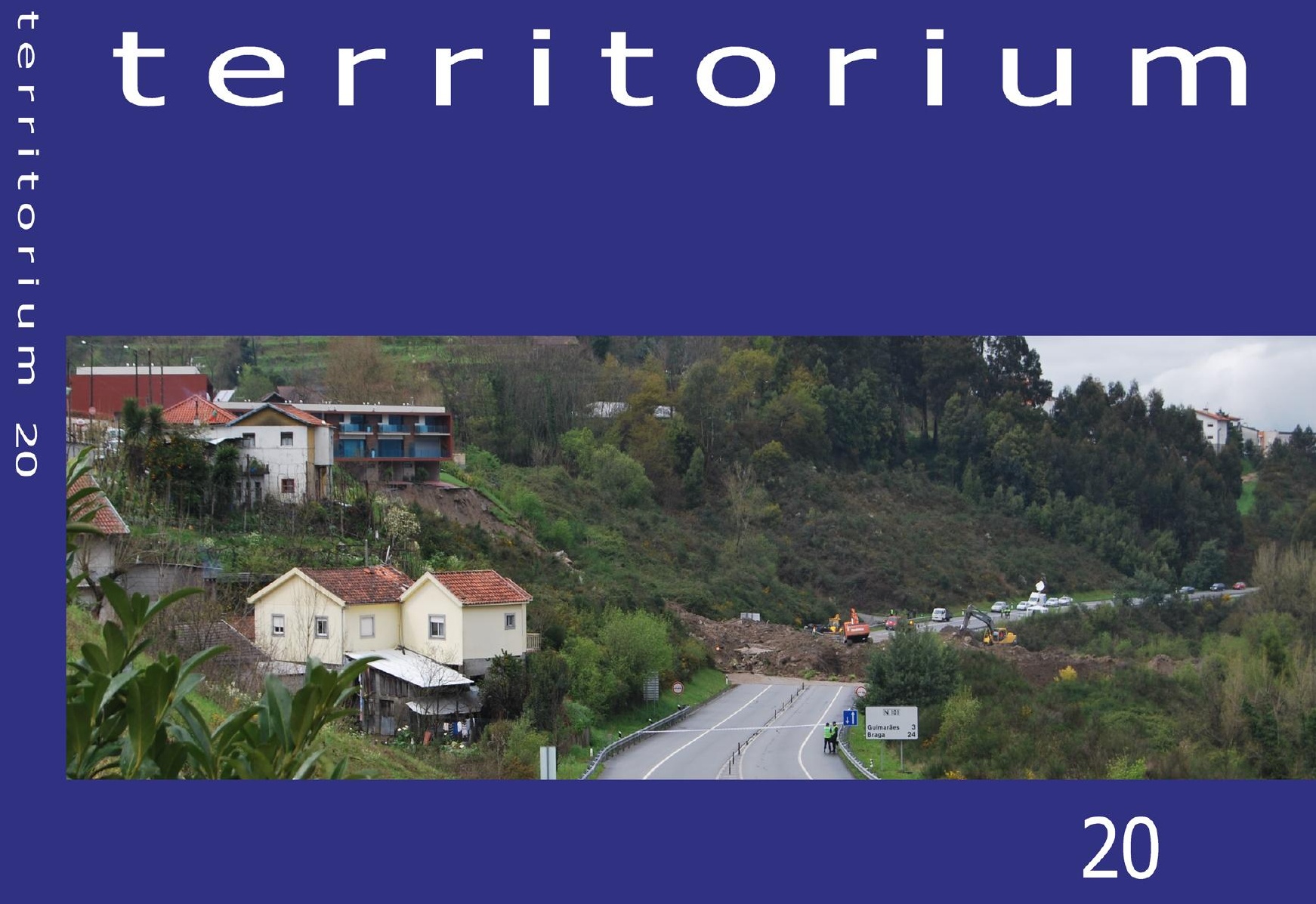

Riscos, População e Segurança 


\section{SISTEMA DE GESTÃO DE RISCOS VIÁRIOS COM O USO DA GEOINTELIGÊNCIA: OS DESLIZAMENTOS E AS INUNDAÇÕES EM RODOVIAS DO ESTADO DE SANTA CATARINA - SUL DO BRASIL*}

Lilian Elizabeth Diesel

Aluna de Pós-Graduação em Geografia Universidade Federal de Santa Catarina lidiesel@gmail.com

\section{RESUMO}

Esta pesquisa apresenta o mapeamento e a aplicação do Raciocínio Baseado em Casos com base em Geointeligência, dos deslizamentos e inundações ocorridos em rodovias do Estado de Santa Catarina, no Sul do Brasil, durante o ano de 2008. Demonstra esta pesquisa que a gestão de risco associada a tecnologias permitem identificar os fenômenos, e as alternativas para as atividades de prevenção, monitoramento, atendimento e resposta.

Palavras-chave: Gestão de risco, geointeligência, SIGRAV.

\section{RESUMEN}

Sistema de gestión de riesgos viarios con uso de la geointeligencia: los deslizamientos y las inundaciones en rutas estaduales de Santa Catarina - sur de Brasil - Esta investigación presenta el sistema de gestión de riesgos en las carreteras, en base a los casos de deslizamientos e inundaciones en las carreteras del Estado de Santa Catarina, en el sur de Brasil. Se exponen, gracias al mapeamiento que permite por medio del RBC, la identificación de los fenómenos y las alternativas para las actividades de gestión, a través de la prevención, el monitoreo, la atención y la respuesta.

Palabras clave: Gestión del riesgo, geointeligencia, SIGRAV.

\section{RÉSUMÉ}

Système de gestion du risque routier avec l'utilisation de geointeligência: les glissements du terrain et les inondations dans les routes de l'état de Santa Catarina, Brésil - Cette recherche présente le système de gestion des risques de voiries/routiers, basée sur les cas de glissements de terrain et inondations sur les routes de l'état de Santa Catarina au Brésil. Sont démontrés, la cartographie qui permet au travers de la réflexion basée sur les faits, l'identification des phénomènes et les alternatives pour les activités de gestion, à travers la prévention, surveillance, accueil et réponse.

Mots-clé: Gestion du risque, géo-intelligence, SIGRAV.

\section{ABSTRACT}

Road risk management system using geointelligence: land slides and floods in the roads of Santa Catarina - southern Brazil - This research presents the risk management system, based on the landslide and floods cases in the roads of the State of Santa Catarina, in southern Brasil. Here are exposed, with the aid of the mapping made possible by the RBC network, the identification of phenomena and alternatives for management activities, through prevention, monitoring, attention and response.

Keywords: Risk management, geointelligence, SIGRAV.

\footnotetext{
* Este artigo é parte integrante da Revista Territorium, $n .{ }^{\circ} 20,2013,{ }^{\circ}$ RIscos, ISBN: 0872- 8941, tendo sido submetido para revisão em 30-07-2012, e aceite para publicação em 12-11-2012.
} 


\section{Introdução}

Os crescentes riscos viários, se não forem geridos com eficiência, podem representar elevados prejuízos sociais, econômicos e ambientais, com reflexos negativos sobre o desenvolvimento sustentável do Brasil. O Sistema de Gestão de Riscos Viários - SIGRAV, usando teorias de Geointeligência (GIS + Al), tem como objetivo o apoio a tomadas de decisões em atividades de prevenção, monitoramento, atendimento e resposta, visando agilizar as diferentes etapas de um processo de gestão de risco para deslizamentos e inundações no sistema rodoviário.

A eficiência na gestão de risco de deslizamentos e inundações no sistema rodoviário depende da complexidade do tema riscos, e da dinâmica do sistema de circulação e transportes de cada região considerada. Em regiões onde o sistema de circulação de transportes atende satisfatoriamente a demanda, a eficiência na gestão de risco é alcançada facilmente. Se essa demanda apresenta um ritmo de crescimento e/ou oscilações, considerado o processo de controle de uso e ocupação do solo, ainda é possível fazer a gestão do risco, desde que se tenham informações em qualidade e quantidade necessárias.

As informações para a gestão de risco devem, no mínimo, permitiridentificar ofenômeno (caracterização, localização, causas) e as alternativas de intervenção. As intervenções se fazem em quatro etapas: prevenção, monitoramento, atendimento e resposta dos danos causados pelos eventos. Estas informações devem estar continuamente atualizadas e disponíveis para diferentes atores, em diferentes formatos e escalas. Devem permitir estas informações, respostas rápidas durante as ocorrências dos eventos e controle das tomadas de decisão e intervenções feitas, agregando novas informações, como subsídios à elaboração de planos de recuperação de áreas afetadas e de planos preventivos para as áreas de risco. São informações de diferentes naturezas, que devem ser constantemente atualizadas e realimentadas, para uso de diferentes atores, para as diferentes etapas do processo de gestão de risco, resultando em grande quantidade de dados a serem processados.

No caso da gestão de risco de desastres nas rodovias, é uma necessidade urgente usar todas as possibilidades para aumentar sua eficácia, em vista das crescentes ameaças em relação às populações humanas e suas atividades. Esse é o avanço da contribuição do conhecimento científico e sua aplicação nas atividades humanas de prevenção a desastres.

\section{Objetivo}

O objetivo desta pesquisa foi o mapeamento dos desastres (deslizamentos e inundações), ocorridos no ano de 2008 e a aplicação do Raciocínio Baseado em Casos (RBC), através do SIGRAV.

\section{Revisão bibliográfica}

A gestão de risco é um tema abordado universalmente por muitos autores, entre os quais se faz destaque a $A$. Lavell (1996, 2000, 2003), O. Cardona (2001), A. Velásquez e C. Rosales (1999), A. Velásquez e N. Jiménez (2004), entre outros. Esses autores conceituam a gestão de risco como um processo composto pela identificação e instrumentação de busca constante de alternativas para a redução dos riscos e dos efeitos de acidentes e desastres já ocorridos, através do planejamento e execução de medidas e ações de prevenção e resposta, articuladas por políticas públicas claras.

A Gestão de risco inicia-se pelo conhecimento do fenômeno (análise) e se realimenta do acompanhamento de sua evolução (monitoramento), para acumular conhecimentos que permitam propor planos que minimizem a evolução do fenômeno.

A. Velásquez e N. Jiménez (2004) afirmam que a gestão de risco é um processo continuo de políticas, medidas e ações conduzindo o controle, e evitando as perdas potenciais por desajustes na interação Meio Ambiente e Sociedade.

Segundo Y. Veyret (2007), o principal componente para a gestão de risco são os atores, e estes se utilizam de conselhos dos especialistas em desastres, especialistas econômicos, da sociedade civil e gestores públicos, para elaborar os alertas, denunciar perigos, estabelecer responsabilidades, e definir dentro de cada situação as frentes que devem ser atacadas. Ainda segundo Y. VeYret (2007), para a gestão de risco é necessário ter o conhecimento da gestão de crise; do acúmulo de experiência; da previsão, prevenção e proteção e por fim da gestão de riscos.

Como ferramenta para a gestão de risco, destacamos o SIGRAV, desenvolvido por L. Diesel (2009), que é um sistema de gestão de riscos viários usando geointeligência para rodovias do Estado de Santa Catarina - Sul do Brasil. Este sistema foi desenvolvido através de Sistemas de Informações Geográficas e Inteligência ArtificialRaciocínio Baseado em Casos (RBC) e teve como objetivo o apoio a tomadas de decisão em atividades de prevenção, monitoramento, atendimento e reposta, visando agilizar as diferentes etapas de um processo de gestão de risco.

A geointeligência segundo sua doutrina básica (2006) tem como definição:

"Exploração e análise da imagem e informação geoespacial para descrever, avaliar e visualizar as feições físicas e as atividades geograficamente referenciadas no mundo. Geointeligência consiste de imagens e informação geoespacial".(NATIONAL GEOSPATIAL-INTELLIGENCE AGENCY. 2006. p.5) 
Ainda conforme a NATIONAL GEOSPATIALINTELLIGENCE AGENCY (2006) a Geointeligência possui a capacidade de realizar analise dinâmica, interativa e simulações, através de mapas interativos, criação de cenários e outros.

Segundo J. Gardner, J. Monget, R. Larsen e T. Warner (2003), a geointeligência é a exploração do banco de dados, imagens e mapas digitais. Ela é aplicada para os recursos naturais, gerenciamento e evolução da economia, petróleo, redes de distribuição de gás, recursos minerais, recursos hídricos, agroindustriais e questões ambientais.

Em concordância com os autores destacados na revisão bibliográfica apontamos que a gestão de risco não deve ser considerada como um conjunto isolado de medidas, e sim de linha de análise e preocupação que atinge toda a atividade humana e possui características que cruzam múltiplas áreas de ação institucional, relacionadas a gestão de recursos naturais, planejamento urbano, regional e setorial, com a gestão da segurança do cidadão, com o desenvolvimento sustentável, entre outras.

\section{Material e método}

Para o estudo da gestão de risco faz-se necessário a realização da revisão bibliográfica, a experimentação, a descrição (estudo de caso, dados históricos), a pesquisa ação e a pesquisa participante. Sendo estes os processos para uma abordagem científica. (E. LaKatos e M. MARCONI 2005, 2006 e 2007; S. Vergara, 2007; R. Richardson, 1989; R. Quivy e L. CAMPENHOUdT, 2005)

Estas abordagens permitem ampliar o horizonte de informações sobre o tema estudado e garantir os enfoques necessários ao alcance de uma maior aproximação da realidade. Nesta pesquisa, optou-se pela pesquisa experimental, com a aplicação do sistema de gestão de risco viário, em rodovias do Estado de Santa Catarina, no Sul do Brasil.

Teve como etapas esta pesquisa a construção da fundamentação teórica, o desenho da pesquisa, a pesquisa de campo e a coleta de dados, a implementação dos dados no SIGRAV e a aplicação experimental dos dados de desastres (deslizamentos e inundações) em rodovias.

\section{A Construção da Fundamentação Teórica}

A etapa de construção da fundamentação teórica foi elaborada através de revisão bibliográfica e documental, através de bibliografias, buscando definições em relação aos desastres naturais, a gestão de riscos, a geointeligência, e seu contexto em Santa Catarina/ Brasil. A partir do que foi levantado, pode-se definir o desenho da pesquisa.

\section{Desenho da Pesquisa}

$\mathrm{Na}$ fundamentação teórica foram levantadas as necessidades de se utilizar ferramentas de gestão, que possam auxiliar os gestores públicos responsáveis pelas atividades de prevenção, monitoramento, atendimento e resposta, em caso de ocorrências de desastres.

Desta forma optou-se para esta pesquisa o mapeamento e as tomadas de decisões, com base em eventos já ocorridos. Para isto, definiu-se como eficaz o uso do SIGRAV, com base em geointeligência.

Pesquisa de campo e coleta de dados

A pesquisa de campo em gestão e risco é uma das recomendações sugeridas por O. CARDONA, (2001), A. Velásquez e N. Jiménez, (2004), A. Velásquez e C. Rosales, (1999), G. Wilches-Chaux, (1998) e Y. Veyret, (2007). Estes autores destacam a necessidade de primeiro conhecer o fenômeno a administrar, antes de conceber um sistema de gestão, devia-se conhecer as organizações, os agentes e as rotinas adotadas para a "gestão de risco", por mais incipiente que seja. Dessa forma, a pesquisa de campo teve dois enfoques: inventário de dados em órgãos públicos e ações de campo acompanhado por agentes públicos.

A pesquisa de campo começou pela aproximação com os órgãos gestores de riscos viários, no Estado de Santa Catarina, e a programação de visitas de campo acompanhando agentes públicos, em atividades de atendimento e definições de intervenções físicas e a caracterização de suas causas. As visitas ocorreram em um número de 8 e deram-se no período de janeiro a dezembro de 2008 e janeiro a junho de 2009.

A consolidação dos conhecimentos adquiridos nas visitas aos órgãos públicos e o acompanhamento de suas atividades no campo permitiu definir as necessidades, em termos de informações para as atividades de gestão de risco e a natureza dos dados, para alimentar o sistema, estes dados são: rodovias, circulação, desastres naturais e características geográficas, como relevo, clima, hidrografia entre outros.

Paralelamente as pesquisas de campo foram sendo inventariadas, paraalimentarosbancosdedadosdoSIGRAV. $\mathrm{O}$ inventário dos dados mostrou a disponibilidade destes em formatos variados. Os dados usados na construção do sistema e suas fontes são relacionados a seguir.

O Estado de Santa Catarina é cortado por oito Rodovias Federais à saber: BR101, BR116, BR153, BR158, BR163, BR280, BR282 e BR470. Estas rodovias somam um total de $2.304 \mathrm{~km}$ 's de extensão. As Rodovias Estaduais no Estado de Santa Catarina tem um total de $3.733 \mathrm{~km}$ 's de extensão e compreendem 95 rodovias. 
No Departamento de Polícia Rodoviária Federal de Santa Catarina (DPRF/SC), Departamento Nacional de Infraestrutura (DNIT) e no Departamento Estadual de Infraestrutura (DEINFRA) foram coletados dados sobre os eventos ocorridos durante o ano de 2008. Esses dados contêm: data, km, metro, localização, rodovia, tipo de localidade, uso do solo, e outros. Todos os dados destacados foram fornecidos em formato digital, arquivo Excel (Microsoft). Na Defesa Civil Estadual, foram coletados dados disponíveis nas Avaliações de Danos (AVADANS), estes não foram disponibilizados em formato eletrônico, sendo necessária a digitação dos mesmos.

A base cartográfica usada para a construção do sistema de gestão, obtida junto ao IBGE, no ano de 2003, em formato digital (shapefile), contendo o canevá cartográfico em coordenadas UTM (Universal Transversal de Mercator), no Sistema de Referência Geodésico SAD69 (South American Datum), com representações equivalentes às escalas de 1:100.000 e 1:50.000. Além do canevá, contém os limites do Estado de Santa Catarina e os limites dos municípios.

O traçado digital das Rodovias Federais e Estaduais no Estado de Santa Catarina foi obtido junto ao DEINFRA, em 26 de março de 2007, também em formato digital (shapefile), com os mesmos referenciais geodésico da base cartográfica.

As cartas digitais de hidrografia, elevação do terreno, relevo, solos, vegetação e curvas de níveis, foram obtidas no Centro Integrado de Meteorologia e Recursos Hídricos/ Centro de Informações de Recursos Ambientais e de Hidrometeorologia de Santa Catarina/ Empresa de Pesquisa Agropecuária e Extensão Rural de Santa Catarina (Climerh/CIRAM/Epagri), em formato digital (shapefile). Também usam coordenadas UTM referenciados ao Datum SAD69. Essas cartas digitais pertencem a Epagri e a SDS (Secretaria de Desenvolvimento Sustentável) e estão protegidas pelo direito autoral brasileiro, nos termos da Lei 9.610/98. Essas cartas encontram-se no site http://ciram.epagri.rct-sc.br:8080/mapoteca/, podendo ser usadas para fins não comerciais, desde que mencionado claramente o direito de propriedade da Epagri e da SDS.

\section{Implementação dos dados no SIGRAV}

A inserção dos dados no SIGRAV foi realizada manualmente, uma vez que os dados disponíveis nas instituições não se encontravam de forma "on line".

Os dados foram inseridos nos bancos de dados pertencentes ao Geodatabase do SIGRAV.

\section{Aplicação Experimental do SIGRAV}

Os riscos viários no caso os deslizamentos e as inundações tratam-se de problemas reais. Sendo problemas reais cabem a eles a aplicação de novos conhecimentos na construção de um modelo de gestão aplicável, cujo funcionamento foi testado através de consultas e análises amostrais. 0 modelo de gestão de riscos foi aplicado através do SIGRAV que alimentado com dados referentes às rodovias do Estado de Santa Catarina e desastres nelas ocorridos, produzem resultados para tomadas de decisões. Os resultados gerados nas análises são armazenados para instruir a memória de conhecimento do sistema e, a partir disso, adotar a técnica do Raciocínio Baseado em Casos (RBC), como apoio à tomada de decisão nas atividades de gestão.

As análises experimentais realizadas pelo SIGRAV são de dois tipos: análises estatísticas e apoio à decisão. A análise de apoio à decisão é realizada usando a técnica de Raciocínio Baseado em Casos (RBC), que pertencente ao ferramental da inteligência artificial (Al). As análises estatísticas são: risco absoluto, risco relativo, coeficiente de morbidade, coeficiente de mortalidade, índices e indicadores de acidentes de trânsito e desastres, em rodovias federais e estaduais de Santa Catarina, por trechos de $1 \mathrm{~km}$ de rodovia. As análises em RBC referem-se à busca por decisões já tomadas em situações anteriores, ou, então, por sugestões de decisão definidas por um especialista, para auxiliar o gestor a tomar as decisões pertinentes a cada situação.

Nesta pesquisa, foram utilizados dados de deslizamentos e inundações em rodovias do Estado de Santa Catarina durante o ano de 2008. No item, resultado encontra-se uma amostra do mapeamento dos desastres e a aplicação da tomada de decisão através do Raciocínio Baseado em Casos, para um evento selecionado.

\section{Resultado}

Como resultado obtido através da aplicação do SIGRAV para deslizamentos e inundações em rodovias, é possível observar na fig. 1, o mapeamento dos eventos ocorridos nos períodos de janeiro/fevereiro de 2008, quando houve excesso de chuva e vários trechos de rodovias ficaram interditados devido a um grande volume de água na pista. No caso de novembro de 2008, além dos volumes de água na pista, ocorreram inúmeros deslizamentos, quedas de barreiras, afundamentos de pista entre outros.

Um dos maiores problemas identificados nas rodovias do Estado de Santa Catarina, encontram-se relacionados aos eventos como os ciclones no Sul do Estado. Na existência deste evento, o Trecho Sul da BR-101 é bastante prejudicado, pois, a rodovia encontra-se praticamente no nível do mar. Desta forma, a presença de água na pista ocorre por dias consecutivos. Uma das ações definidas pelo Raciocínio Baseado em Casos (RBC) seria a rota alternativa, e a implementação de um sistema de drenagem eficaz, para que os usuários, principalmente os caminhões, evitem a paralisação por dias consecutivos. 


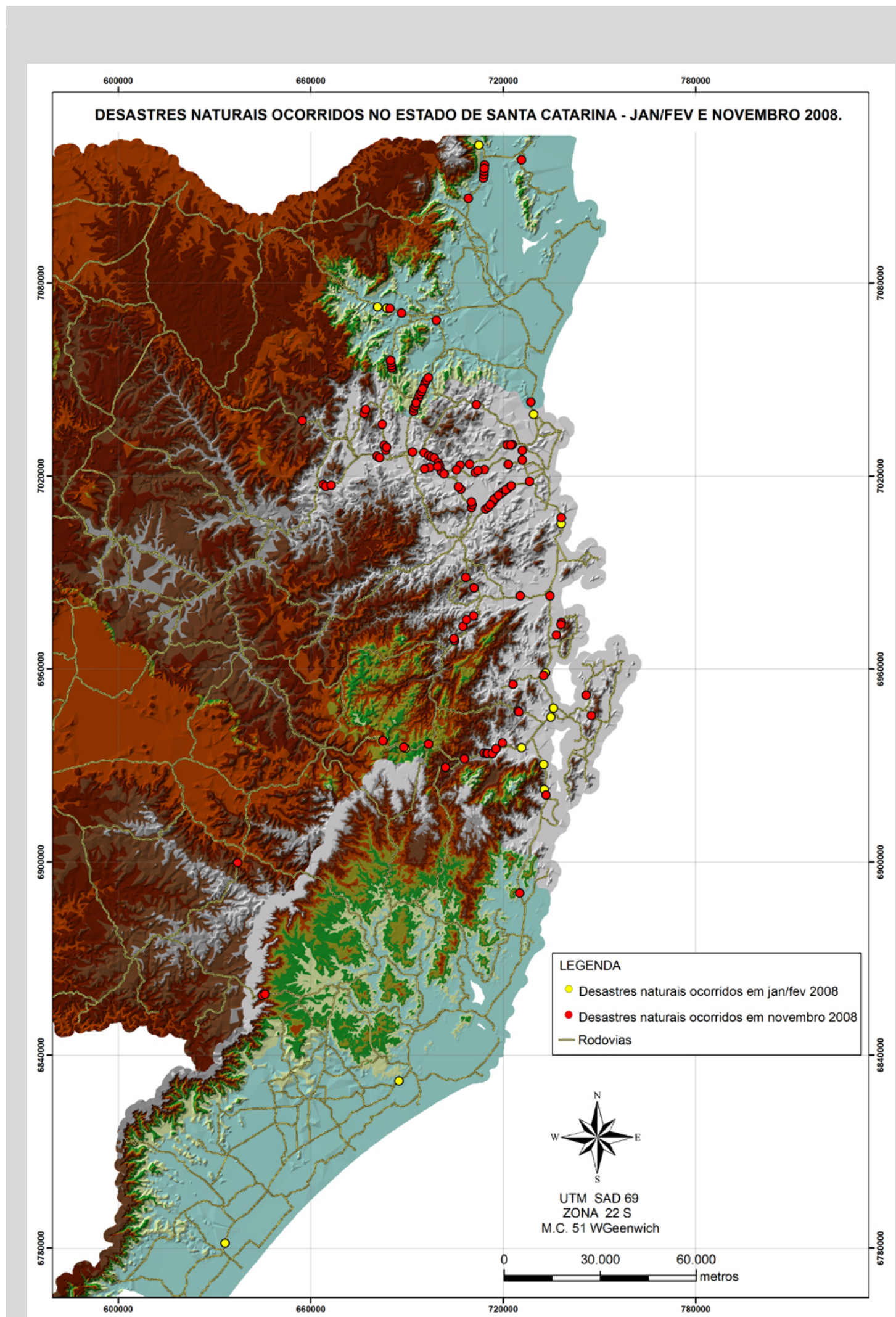

Fig. 1 - Desastres naturais ocorridos no Estado de Santa Catarina - janeiro/fevereiro e novembro 2008. Fonte: L. DIesel, 2009. 
Após o mapeamento dos eventos (fig. 1), foi aplicada a consulta ao RBC, que é realizada através da busca automática das áreas definidas pelo especialista/ usuário. Neste caso, foi selecionado o km 166 da BR101, no município de Tijucas/SC, como área definida para a consulta ao RBC. O RBC lista todas as variáveis correspondentes ao $\mathrm{km}$ selecionado e estas são classificadas em um conjunto de valores (TABELA I).

Com base na pergunta (informações sobre o km $166 \mathrm{da}$ BR-101/SC) enviada ao RBC, analisam-se as variáveis de indexação de casos. No momento da pesquisa, analisa-se a eficácia do sistema através de uma analise de acuracia realizada pelo especialista, e, desta forma, temos a construção do caso (fig.2)

\begin{tabular}{|c|c|c|}
\hline \multirow[b]{2}{*}{ C } & \multicolumn{2}{|l|}{ PROBLEMA } \\
\hline & $B R$ & 101 \\
\hline \multirow{2}{*}{ A } & $\mathrm{Km}$ & 166 \\
\hline & Tipo de desastre & Pista alagada \\
\hline S & Número de repetições & 1 \\
\hline \multirow{2}{*}{0} & Uso do solo & Rural \\
\hline & Localidade & Não edificada \\
\hline \multirow{4}{*}{1} & Traçado & Reta \\
\hline & Condições da pista & Bom \\
\hline & \multicolumn{2}{|l|}{ SOLUÇÃO } \\
\hline & Ações & Drenagem da pista \\
\hline
\end{tabular}

Fig. 2 - Exemplo da construção de um caso pelo RBC, para desastre ocorrido no Km166 da BR-101. Fonte: SIGRAV - módulo RBC.

O RBC lista ainda outras possíveis soluções para o presente caso (fig. 3).

\begin{tabular}{|l|}
\hline Ações Sugeridas \\
\hline Drenagem na pista \\
\hline Mudanças de rotas - caminhos alternativos \\
\hline Proteção das encostas \\
\hline Duplicação da pista \\
\hline Sinalização para queda de barreiras \\
\hline
\end{tabular}

Fig. 3 - Universo de possíveis soluções apontadas pelo grupo multidisciplinar de especialistas em riscos viários. Fonte: SIGRAV - módulo RBC.
Portanto, para a gestão de risco de desastre, o RBC indica as possíveis soluções: drenagem na pista e mudanças de rotas - caminhos alternativos -. Estas possíveis soluções podem ser trabalhadas de forma preventiva, antes de um novo acontecimento, como, também, para medidas emergenciais.

No caso da gestão de riscos, os especialistas têm condições de realizar rotineiramente estas consultas e inserir novas possibilidades para tomadas de decisões, assim como, elencarem medidas de intervenções a serem executadas a médio e longo prazo, objetivando a minimização destas ocorrências.

\section{Conclusões}

A presente pesquisa foi motivada pela gravidade dos problemas de circulação viária no Brasil. Sendo um país de dimensões continentais e concentração quase absoluta da circulação no modal rodoviário, os desastres naturais com impactos sobre a rede viária, tem despertado a atenção de estudiosos, de gestores públicos e de políticos brasileiros. Os crescentes riscos, se não forem geridos com eficiência, podem representar altos prejuízos sociais, econômicos e ambientais, com reflexos negativos sobre o desenvolvimento sustentável do país.

O direcionamento da pesquisa é a busca por caminhos que auxiliem na compreensão do problema e apontem alternativas de melhorias. Partindo da premissa de que os riscos podem ser geridos, através das seguintes etapas prevenção, monitoramento, atendimento e resposta através da associação dos aspectos geográficos aos tecnológicos, em sua estrutura de variáveis.

No Brasil, todos os estímulos são importantes na busca de maior eficácia na gestão territorial. Os problemas têm crescido no mesmo ritmo do crescimento do país e a tradição da gestão “amadora”, principalmente nas questões de competência pública, ainda está bem presente na realidade brasileira.

A gestão de riscos viários é um estímulo para uma efetiva gestão de riscos nas rodovias do Estado de Santa Catarina.

TABELA I - Variáveis e valores do RBC, para desastre ocorrido no Km166, da BR101.

\begin{tabular}{|c|c|c|c|c|}
\hline Número de faixas & Fluxo & Localidade & Tipo de solo & Traçado \\
\hline 1 faixa & Intenso & Comercial & Urbano & Cruzamento \\
\hline 2 faixas & Moderado & Residencial & Rural & Reta \\
\hline 3 faixas & Baixo & Industrial & Urbano e Rural & Curva \\
\hline 4 faixas & & Não edificada & & Reta e curva \\
\hline & & Comercial e Residencial & & \\
\hline & & Comercial e Industrial & & \\
\hline & & Comercial, Residencial e Industrial & & \\
\hline
\end{tabular}


No entanto, essa pesquisa é apenas o início de um grande desafio para o aprimoramento da gestão de "riscos viários". Muito trabalho ainda há a fazer, por diferentes profissionais, de várias áreas do conhecimento.

Para finalizar, é possível afirmar que no decorrer da análise aqui realizada, observou-se que a gestão de risco associada a tecnologias permitem identificar os fenômenos, e as alternativas para as atividades de prevenção, monitoramento, atendimento e resposta.

\section{Referências Bibliográficas}

Cardona, O. M. A. (2001) - Estratégia de Divulgación e Información para la Gestión de Riesgos. LA RED. Santo Domingo, 58 p.

Diesel, L. E.. (209) - Proposta de um sistema de gestão de riscos viários (SIGRAV/2009) usando geointeligência para rodovias do Estado de Santa Catarina - Sul do Brasil. XVI, 186 f. Tese (Doutorado) - Universidade Federal de Santa Catarina, Centro Tecnológico, Programa de Pósgraduação em Engenharia Civil, Florianópolis.

Gardner, J. V.; Monget, J. M.; Sinding- LarSen, R.; Warner, T. (2003) - Geointelligence for assessing natural resource project risks. Geoscience and Remote Sensing Symposium. IGARSS apos; 03. Proceedings. 2003 IEEE International Volume 7, Issue, 21-25 July 2003 Page(s): 4555 - 4557 vol.

Lakatos, E. M., Marcon, M. de A. (2005) - Fundamentos de metodologia científica. 6. Ed. São Paulo: Atlas, 2005. 315p.

Lakatos, E. M., Marcon, M. de A. (2005) - Metodologia cientifica: ciência e conhecimento científico, métodos científicos, teoria, hipóteses e variáveis. 4. ed. São Paulo: Atlas, 2006. 305p. ISBN

Lakatos, E. M., Marcon, M. de A. (2007) - Metodologia cientifica: ciência e conhecimento cientifico, métodos científicos, teoria, hipóteses $e$ variáveis. 5. ed. rev. e ampl. São Paulo: Atlas, 312p.

LAVELL, A. (1996) - Degradación ambiental, riesgo y desastre urbano. Problemas y conceptos: hacia la definición de una agenda de investigación. In. FERnÁndeZ, M.A. ciudades en riesgo degradación ambiental, riesgos urbanos y desastres. La rede - Red de Estudios Sociales en Prevención de Desastres en América Latina.

LavelL, A. (2003) - Gestión local del riesgo: Nociones y precisiones en torno al concepto y la práctica. Centro de Coordinación para la Prevención de los Desastres Naturales en América Central (CEPREDENAC);PNUD; 2003. 101 p. ilus.

LAVELL, A. (2000) - Marco conceptual y analítico programa de vulnerabilidad de el Bajo Lempa Prevención y mitigación de desastres naturales. Proyecto MARN-BID.

NATIONAL GEOSPATIAL-INTELLIGENCE AGENCY (2006) National System for Geospatial Intelligence Geospatial Intelligence (GEOINT) Basic Doctrine, Publication 1-0. http://www.nga. mil/NGASiteContent/StaticFiles/OCR/geo_ pub1.pdf

Quivy, R., CAmPENHoudt, L. (2005) - Manual de investigação em ciências sociais. 4. ed Lisboa: Gradiva, 282p. (Trajectos, 17)

Richardson, R. J. (1989) - Pesquisa Social - Métodos e Técnicas. São Paulo: Atlas.

Velásquez, A.; Jiménez, N. (2004) - La gestión de riesgos en el ordenamiento territorial: inundaciones en cali, la c.v.c y el fenómeno enso. Disponível em:http: / / osso.univalle.edu.co/doc/ congresos/2004/A_Velasquez_Articulo_OSSOUV.pdf Acessado em: 23/03/2007 as 20:08hs

Velásquez, A.; Rosales, C. (1999) - Escudriñando en los desastres a todas las escalas. Concepción, metodología y análisis de desastres en América Latina utilizando DesInventar. OSSO / ITDG / LA RED. Colombia.

Vergara, S. C. (2007) - Projetos e relatórios de pesquisa em administração. 9. ed São Paulo: Atlas, 92p.

Veyret, Y. (2007) - Os Riscos - o Homem como Agressor e Vítima do Meio-ambiente. São Paulo, ed. Contexto.

Wilches Chaux, G. (1998) - Auge, caída y levantada de Felipe Pinillo, mecânico y soldador. Guía de La Red para la gestión local de los desastres. La Red, Quito, Ecuador, 103 p. 